\title{
The Effectiveness of Toxoplasma gondii Infection in the Emotions of Pregnant Women in Makkah, Saudi Arabia
}

\author{
Khalil Mohamed $^{\mathrm{a}}$, Adil Bahathiq ${ }^{\mathrm{b}}$, Narjes Degnah ${ }^{\mathrm{c}}$, Mohamed Abdel-fattah ${ }^{\mathrm{a}, \mathrm{d}}$, Suzan Basuni ${ }^{\mathrm{e}}$, Al Bagir Mahdi ${ }^{\mathrm{f}}$, \\ Hosham Karar ${ }^{\mathrm{g}}$, Ahmad Babalghith
}

${ }^{a}$ Department of Epidemiology, Faculty of Public Health \& Health Informatics, Umm Al Qura University.

${ }^{b}$ Department of Physiology, Faculty of Medicine, Umm Al Qura University.

${ }^{c}$ Department of Laboratory, Maternal and Child Hospital. Ministry of Health.

${ }^{d}$ Research and Development Department, VACSERA, Egypt

${ }^{e}$ Department of Psychiatric, Faculty of Education, Umm Al Qura University.

${ }^{f}$ Department of Obstetrics \& Gynaecology, Faculty of Medicine, Umm Al Qura University.

${ }^{g}$ Department of Promotion \& Health Education, Faculty of Public Health \& Health Informatics, Umm Al Qura University.

${ }^{h}$ Department of Medical Genetics, Faculty of Medicine, Umm Al Qura University.

Correspondence Author: Khalil Mohamed, Department of Epidemiology, Faculty of Public Health \& Health Informatics, Umm Al Qura University. E-mail:- kmismail@uqu.edu.sa; khali72@gmail.com

Received date: 11 December 2018, Accepted date: 22 Janaury 2018, Online date: 29 January 2019

Copyright: () 2019 Khalil Mohamed et al, This is an open-access article distributed under the terms of the Creative Commons Attribution License, which permits unrestricted use, distribution, and reproduction in any medium, provided the original author and source are credited.

\begin{abstract}
The parasite causes toxoplasmosis in human and animals are Toxoplasma gondii which is found in the third of human population around the world. T. gondii is an obligate protozoan which parasitizes all warm-blooded animals including human. The effectiveness of the latent parasite to the people particularly in the personality characteristics or behaviour of latent infective people. The current study is performed to explain the effectiveness of latent toxoplasmosis in the emotions of pregnant women.

A case-control study is performed to explain the effectiveness of infection by $T$. gondii to the emotions of pregnant women. Data was collected by using questionnaire after all subjects signed the consent form. Latent infection differentiated by using ELISA IgG and IgM.

In the first dimension, the results showed the relationship between latent toxoplasmosis and feeling pessimistic about the future from the side and feel not qualified in the life from other hand $(p<0.05)$. In dimension two, there was closed relation between latent infection and patient never offer a benefit as in previously, and also we found a correlation between the latent toxoplasmosis and not thinking in suicide $(p<0.05)$. In the dimension three, there was an association between infections with latent toxoplasmosis and insomnia $(p<0.05)$. The correlation between the efficiency of concentration and infected with $T$. gondii observed in this study $(p<0.05)$ also, the results found there was relationship between exhausted and infection with the parasite in dimension four $(p<0.05)$.

In conclusion, the present study presented the impact of latent toxoplasmosis to the pregnant women which included feeling pessimistic about future, feeling not qualified in the life, and feeling has no benefit from her life.
\end{abstract}

Key words: Latent, chronic, toxoplasmosis, emotions, pregnant, and Makkah.

\section{INTRODUCTION}

The parasite causes toxoplasmosis in human and animals are Toxoplasma gondii which found in the third of human population around the world. T. gondii is an obligate protozoan which parasitizes all warm-blooded animals including human (Liu et al., 2012; Liu et al., 2015). The primary infection in human may happen by direct contact with the faces of an infected domestic cat which play as the main definitive host where the parasite can complete the life cycle (Robert-Gangneuxa \& Dardéc, 2012; Sugden et al., 2016). An indirect source of infection occurs when consumption infected raw meat or exposure to soil or drinking water contaminated with the oocytes of the parasite or vertical transmission from mother to her foetus during pregnancy (RobertGangneuxa \& Dardéc, 2012; Sugden et al., 2016).

The symptoms of infection with $T$. gondii in pregnant women vary according to the stage of pregnancy. Once a pregnant woman got the infection during pregnancy serious symptoms may occur such as neurological and prematurity, disorders of ophthalmic, restricted intrauterine growth, and miscarriage, (Jones et al., 2003; Pessanha et al., 2011). 
Globally, the status of disease vary from area to another area due to different reasons, in Southern European countries the seroprevalence may reach 54\% (Cook et al., 2000). While the prevalence may become high in Sub-Saharan countries where 92.5\% has been recorded (Ayi et al., 2009), and 70\% in Southeast Asian countries (Nissapatorn, 2007). The maximum prevalence rate in Saudi Arabia had recorded 38\% (Almogren, 2011); the prevalence rate in pregnant women in Makkah had reached 35.6\% (Al-Harthi et al., 2006).

Detection of $T$. gondii infection in pregnant women based on presented $\operatorname{IgG}$ and $\operatorname{IgM}$ to confirm the infection for their sensitivity and specificity (da Silva et al., 2015).

After the recent infection by $T$. gondii, the parasite may dormant in the brain and muscle of infected people without appears of obvious symptoms. This phenomenon called latent toxoplasmosis. Several studies were done in different countries in different target groups to explore the effectiveness of the latent parasite to the people particularly in the personality characteristics or behaviour of latent infective people. These studies found correlation between latent toxoplasmosis and schizophrenia (Torrey et al., 2007) and Parkinson's disease (Miman et al., 2010) inspirations of personality and behaviour of human (Arling et al., 2009) impairs performance of psychomotor, suicide risk enhancement (Flegr et al., 2002), causes the traffic accident (Yereli et al., 2006; Kaňková et al., 2007a; Kocazeybek et al., 2009; Flegr et al., 2009), and promotions the probability of delivery male offspring (Novotná et al., 2005; Kan̆ková et al., 2007b).

The effectiveness of latent toxoplasmosis infection in men found to be more likely to ignore rules and was more expedient, jealous, dogmatic, and suspiciously. While the effectiveness made women more likely warm-hearted, moralistic, persistent, conscientious, and outgoing (Flegr. 2007).

The main aim of the current study is performed to explain the effectiveness of latent toxoplasmosis in the emotions of pregnant women.

\section{MATERIAL AND METHODS}

\section{Study Design}

The current study was the case-control to compare between two target groups. The first group was pregnant women in different trimesters of pregnancy with latent toxoplasmosis, and the second group was pregnant women also in various stages of pregnancy without $T$. gondii infection.

\section{Data Collection and Consent}

Information from participants was collected using questionnaire after obtained their agreement and signed the consent. The interview was done with each participant before sample collection.

\section{Sampling Process}

The blood sample from vein collected from each pregnant woman participated in the current study. Blood samples were put in the EDTA tubes and centrifuged at 5,000g for 10 minutes. Plasma separated and transferred into cryotube and stored at $-80^{\circ} \mathrm{C}$ until analysed.

\section{ELISA IgM and IgG}

ELISA IgM and IgG was performed to detect anti-Toxoplasma antibodies and to differentiate between recent infection and latent toxoplasmosis. In ELISA procedures, researchers follow the manufacturing instructions (Humans $®)$.

\section{Data Analysis}

The data analysed by using SPSS packages version 20. Categorical variables expressed as percentages and the continuous variables as form of means \pm standard deviations. We used $C h i$-square test to comparison the frequencies among groups. $P$-value considered statistically as $<0.05$ for significant.

\section{RESULTS AND DISCUSSION}

\section{Study population}

A total of 339 pregnant women participated in this study in different trimesters of pregnancy. To measure the effectiveness of latent toxoplasmosis to the behaviours of pregnant women, a list of questionnaire used, and all the participants provided blood sample to determine $\operatorname{IgG}$ and IgM concentrations for $T$. gondii using ELISA test. The most common protozoan parasite in developed countries is T. gondii. The parasite assumes a latent form after the early acute infection. Approximately, $80 \%$ of the population may be infected, due to eating habits or exposure to infected cats (Tenter et al., 2000). The latent form of T. gondii is found predominantly in muscle and nervous tissues in infected host. Until recently, latent toxoplasmosis in man was considered to be asymptomatic.

\section{Seroprevalence using ELISA IgG \& IgM}

To differentiate between recent and latent infection with toxoplasmosis, ELISA IgM and IgG are performed to detect antiToxoplasma antibodies in the sera provided by the pregnant women. A total of 326 out of 339 plasma samples from women examined by using ELISA IgG while 301 out of 339 examined by using ELISA IgM. The results presented in table (1) indicated a total prevalence rate of $67(19.8 \%)$ and $2(0.6 \%)$ of women showed latent and recent infection by $T$. gondii, respectively. 
Citation: Khalil Mohamed, et al., The Effectiveness of Toxoplasma gondii Infection in the Emotions of Pregnant Women in Makkah, Saudi Arabia. Australian Journal of Basic and Applied Sciences, 13(1): 1-8. DOI: 10.22587/ajbas.2019.13.1.1

Table (1): Prevalence of $\operatorname{IgM}$ and $\operatorname{IgG}$ in pregnant women

\begin{tabular}{|c|c|c|c|c|}
\hline & \multicolumn{2}{|c|}{ IgM } & \multicolumn{2}{c|}{ IgG } \\
\hline & Frequency & Percent & Frequency & Percent \\
\hline Negative & 299 & $88.2 \%$ & 259 & $76.4 \%$ \\
\hline Positive & 2 & $0.6 \%$ & 67 & $19.8 \%$ \\
\hline Total (Valid) & 301 & $88.8 \%$ & 326 & $96.2 \%$ \\
\hline Missing & 38 & $11.2 \%$ & 13 & $3.8 \%$ \\
\hline Total & 339 & $100 \%$ & 339 & $100 \%$ \\
\hline
\end{tabular}

The results obtained from this study divided into two parts; questionnaire survey and serological assays; ELISA for IgG and IgM. Detection of IgG in blood samples was for previous infection and IgM for recent infection in pregnant women. Prevalence of previous T. gondii infection among pregnant women in Makkah City in the current study was $19.8 \%$ which is familiar to previous studies done in the same area, and same target group recorded 35.6\% IgG seropositivity by using ELISA (Ghazi et al., 2002). AlHarthi et al., (2006) in the same target group showed that the prevalence was $29.4 \%$ and by using ELISA for IgG have shown that the prevalence using ELISA IgG was lowered to be $21.2 \%$ (Khalil et al., 20016).

Comparing the results obtained in the current study with previous studies done in the same area, it noticed remarkably that the prevalence of $T$. gondii among pregnant women in Makkah City is continued to be much lowered. This lowering in prevalence may refer to different factors such as 1-improvements in healthy habits, 2-change in environmental factors favouring the transmission and infectivity of $T$. gondii oocysts, 3-increase of health awareness, 4-change in living styles or 5- getting good knowledge about source of $T$. gondii infections.

\section{Questionnaire survey}

To determinate the exposure of participants to the emotional factors associated with the latent $T$. gondii infection, pregnant women were asked 53 questions about five types of dimensions deal with the behavioural and emotional characteristics. Each question answered by three choices (Agree, Do not agree, to some extent). The participants' answers are divided according to IgG and IgM ELISA results as follows:

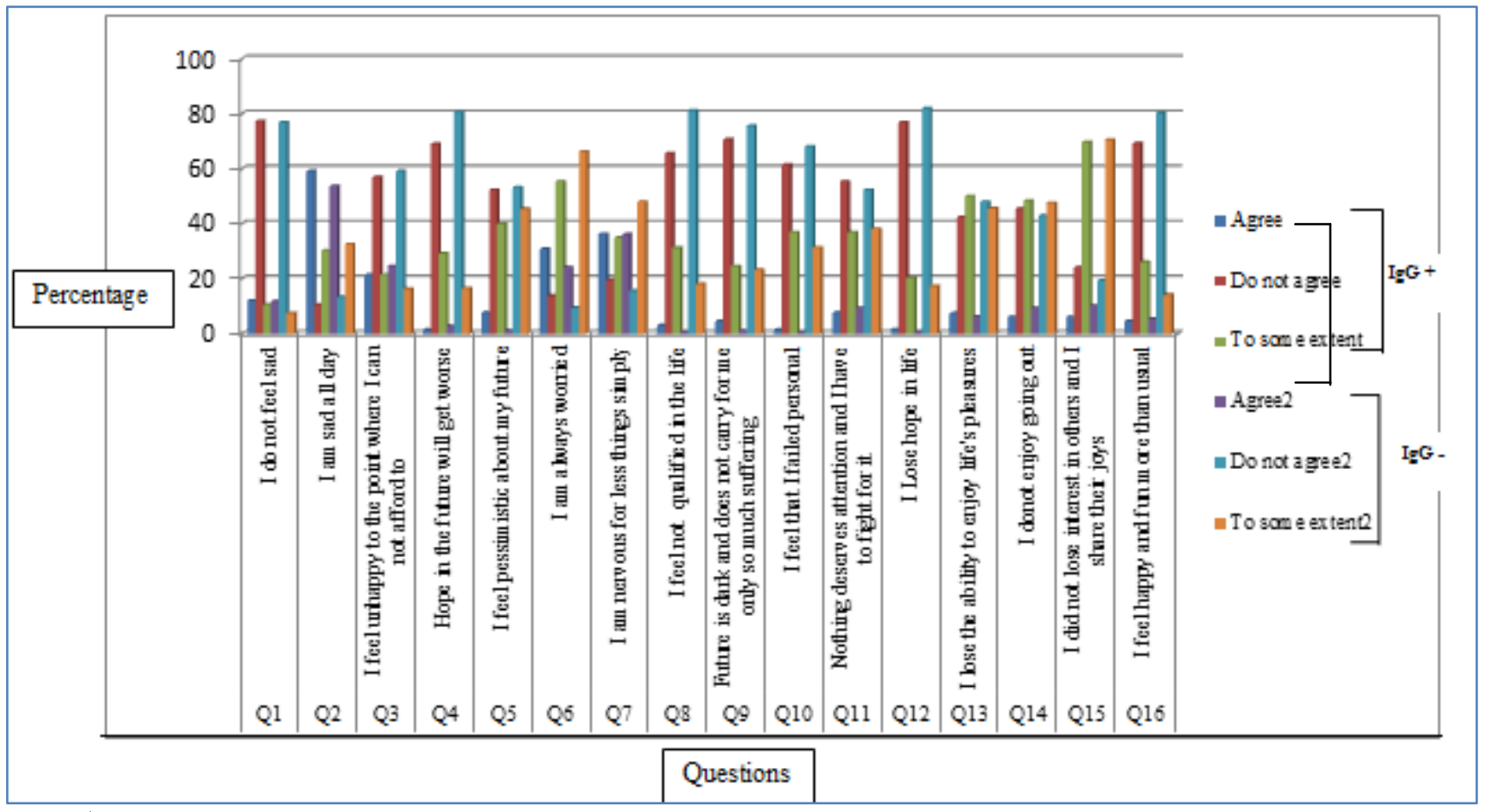

Figure $1.1^{\text {st }}$ dimension: sadness and lack of sense of life quality (no. of questions 16) (Figure 1).

Figure 1: Shows the percentages of the different answers of the first dimension among IgG positive and IgG negative ELISA results. The results of dimension 1 showed that 14 out of 16 questions were with no relation with the latent T. gondii ( $p$ $>0.05$ ), while Question \# 5 (I feel pessimistic about my future) and question \# 8 (I feel not qualified in the life) showed a close relationship with the toxoplasmosis IgG positive ELISA group $(p<0.05)$.

The questionnaire is one of the essential strategies that used in studying the relation between the latent toxoplasmosis and different factors such as environmental factors, personality changes, human behavioural factors (Flegr et al., 2000; Flegr, 2007) .

In this study, the questionnaire survey was used to measure the emotional variations among the infected subjects. Our results showed that seven questions have showed a close relation with latent toxoplasmosis infection $(p<0.05)$ which reflect a significant measure for the relation between emotional variations in pregnant women and infection with latent toxoplasmosis. 
It is well known that a number of parasites make personality changes in their hosts' behavior. Latent toxoplasmosis in humans is associated with personality changes profiles (Flegr et al., 2000). In the latent toxoplasmosis, the parasite changes the form to bradyzoites and can survive in the infected subject for life in muscular and neural tissues of the host (Remington \& Krahenbuhl, 1982). Latent toxoplasmosis can reactivate into acute toxoplasmosis due to defect in the immune system such as infected with AIDS or using immunosuppressive drugs, etc. (Mocsny, 1992; Heitman \& Irizarry, 1997).

The results found during the past 25 years supported the suggestion that latent toxoplasmosis has influences in the behavior of the host not only in rodent hosts but also of humans. The reasons and the mechanisms and the effectiveness of these behavioural changes, however, are still to be explained (Flegr, 2007). Yet, important changes in personality profiles of women and men with latent toxoplasmosis and healthy controls were occurred and reported (Flegr \& Hardy, 1994; Flegr et al., 1996). In women with latent toxoplasmosis they found that they had higher ergic tension and radicalism, guilt proneness (are self-reproaching, more apprehensive, insecure), and higher intelligence (Flegr \& Havlicek, 1999).

In the current study, the questionnaire results suggested a positive correlation between latent toxoplasmosis and change in personality and behavior as the three questions: I can never offer a benefit as I was previously; I cannot concentrate on the same efficiency; I'm very exhausted have supported significantly such correlation $(p<0.05)$.

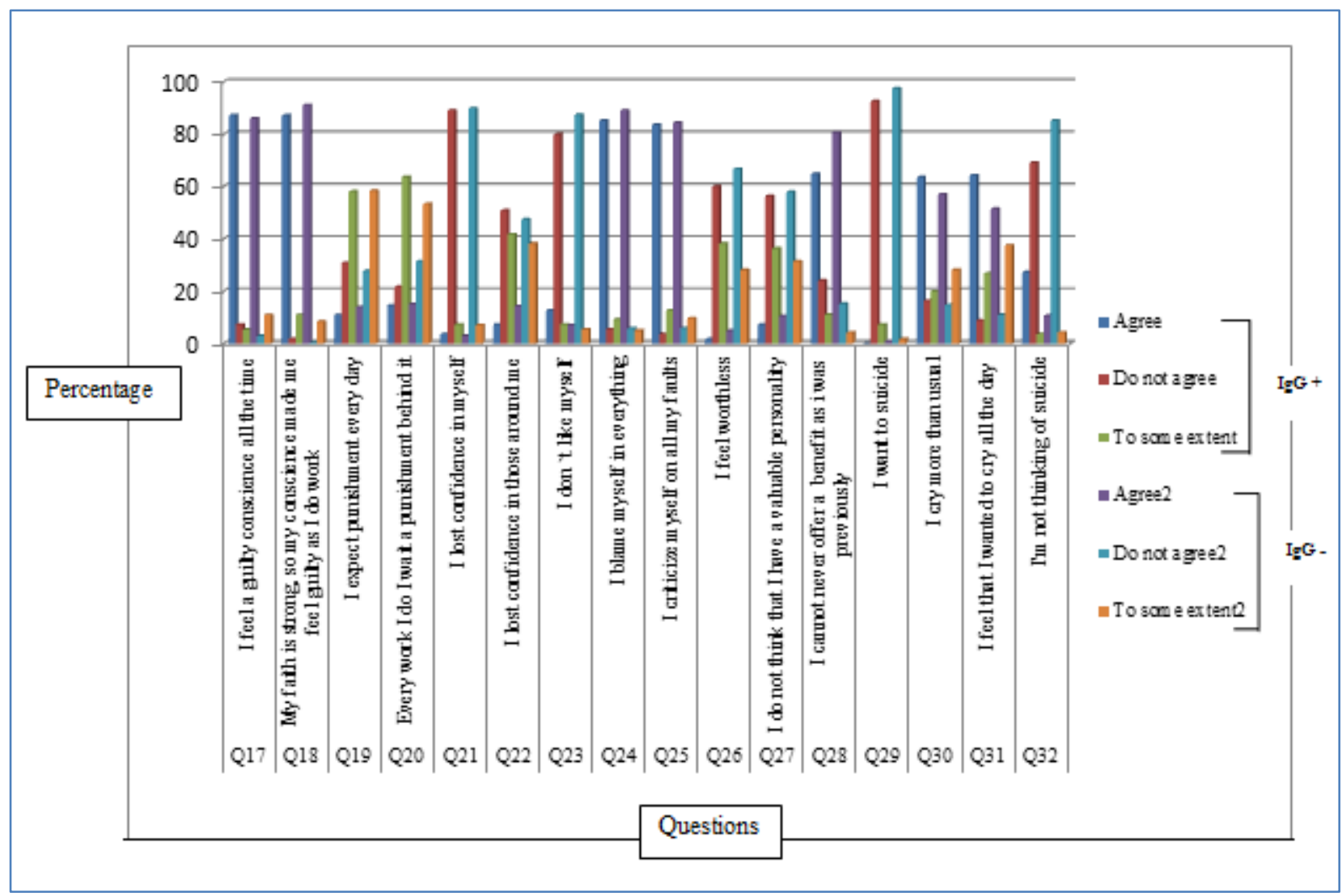

Figure $2.2^{\text {nd }}$ dimension: Self-criticism and lack of sense of value (no. of question 16).

Figure 2 shows the percentages of the different answers of the second-dimension questions among IgG positive and IgG negative ELISA results. The results of dimension two showed that 14 out of 16 questions were with no relation with the latent $T$. gondii ( $p$ $>0.05$ ), while question \# 28 (I can never offer a benefit as I was previously ) and question \# 32 (I'm not thinking of suicide) showed a close relationship with the toxoplasmosis IgG positive ELISA group $(p<0.05)$. Alvarado-Esquivel et al., (2016) assessed the association of $T$. gondii infection and depression in a sample of psychiatric patients and control subjects without depression. The results of their study suggested a potential relationship between infection with $T$. gondii and depression. It is well known that high titters of $T$. gondii IgG in infected women were correlated to depression and anxiety during pregnancy. Reactivation of latent toxoplasmosis may alter mood in pregnant women (Groër et al., 2011). On the other hand, the association of T. gondii IgG with depression and anxiety in pregnancy is important for a number of reasons. Over one-third of the population around the world have antibodies against $T$. gondii (Montoya \& Liesenfeld, 2004). Prenatal depression and anxiety are also common and associated with adverse pregnancy and foetal outcomes (Bansil et al., 2010). It is also a predictor of postpartum depression.

The preliminary work was done by Groër et al., (2011), have suggested that there are relationships between depression, immunity, T. gondii IgG titers and cytokines in pregnancy that may translate into significant health risks for both mother and foetus. Infection with $T$. gondii parasites may also lead indirectly to anxiety-like behaviours, via the response of host immunity. Cytokine production plays a key role in sustaining the bradyzoites of the latent toxoplasmosis (Carruthers \& Suzuki, 2007).

In the current study, our results agree with such correlation and the questions: I feel pessimistic about my future; I feel not qualified in the life to have showed a positive relation with participants women infected with latent $T$. gondii ( $p<0.05)$. Also, our results have showed that none of the cases seropositive to $T$. gondii had IgM antibodies, suggesting that depression and anxiety 
Citation: Khalil Mohamed, et al., The Effectiveness of Toxoplasma gondii Infection in the Emotions of Pregnant Women in Makkah, Saudi Arabia. Australian Journal of Basic and Applied Sciences, 13(1): 1-8. DOI: 10.22587/ajbas.2019.13.1.1

might be associated with latent infection. This association may be explained as a result of tachyzoites of $T$. gondii invade some of cells in brain (Fond et al., 2013), and it is possible that T. gondii causes mood disorders through changes in the levels of brain dopamine and serotonin (Groër et al., 2011).

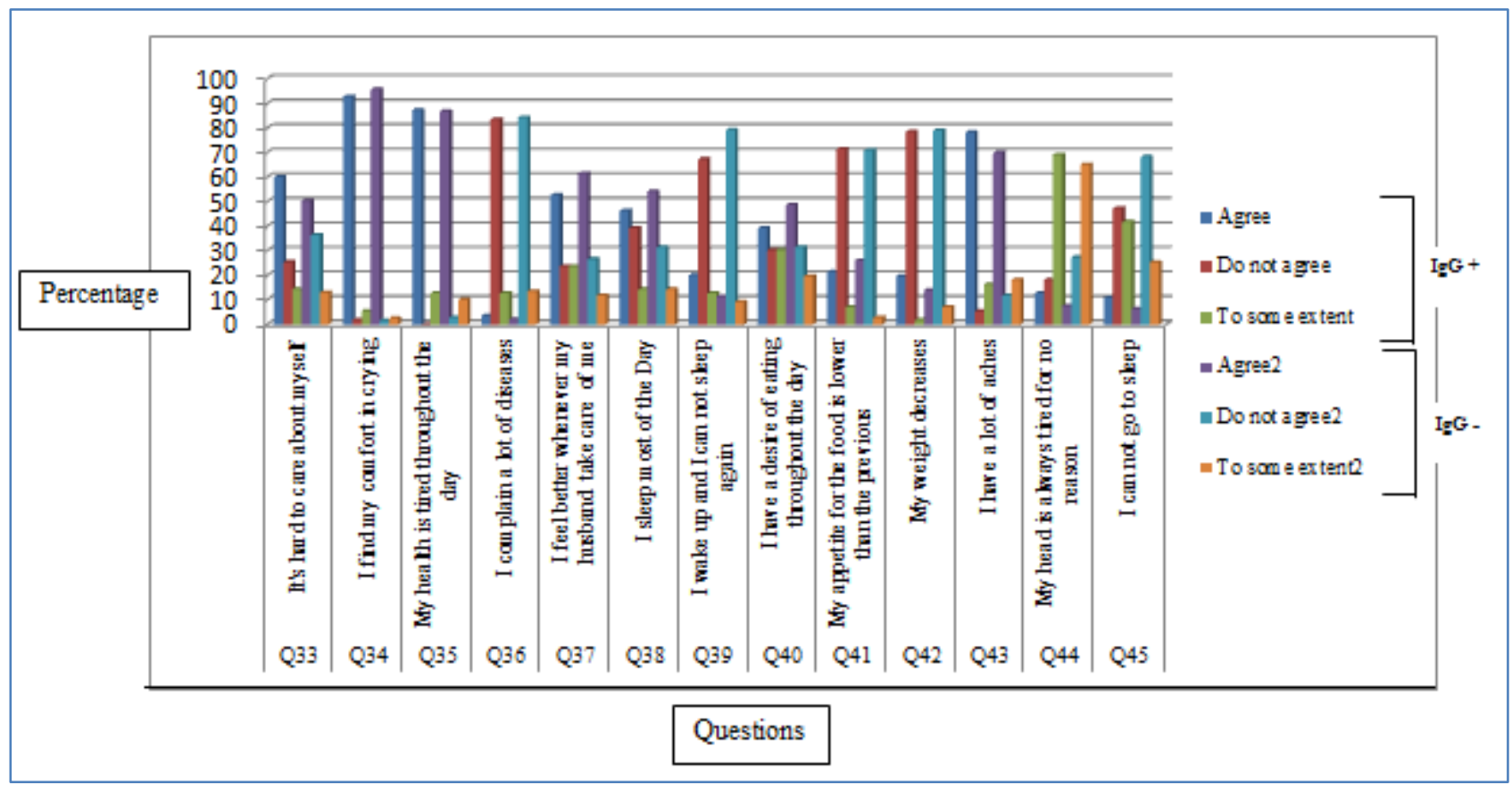

Figure 3. $3^{\text {rd }}$ dimension: Physical and health disorders (no. of question 13).

Figure 3 shows the percentages of the different answers of the third-dimension questions among IgG positive and IgG negative ELISA results.

The results of dimension three showed that 12 out of 13 questions were with no relation with the latent T. gondii $(p>0.05)$, while question 45 (I cannot go to sleep, insomnia) showed a close association with the toxoplasmosis IgG positive ELISA group $(p<0.05)$. Timothy et al., (2009) hypothesized that seropositive against $T$.gondii will be connected with suicide attempted. Their opinion confirmed that people with high intensity of antibodies against $T$. gondii will be found among suicide attempters than nonattempters. Also the history of suicide attempts has widely studied (Arling et al., 2009; Yagmur et al., 2010; \& Ling et al., 2011). Changed serotonergic purpose, in addition to its relationship with aggression (suicide risk factors), impulsivity, and depression, has been dependably reported to be related with behaviour of suicidal (Mann, 2003).

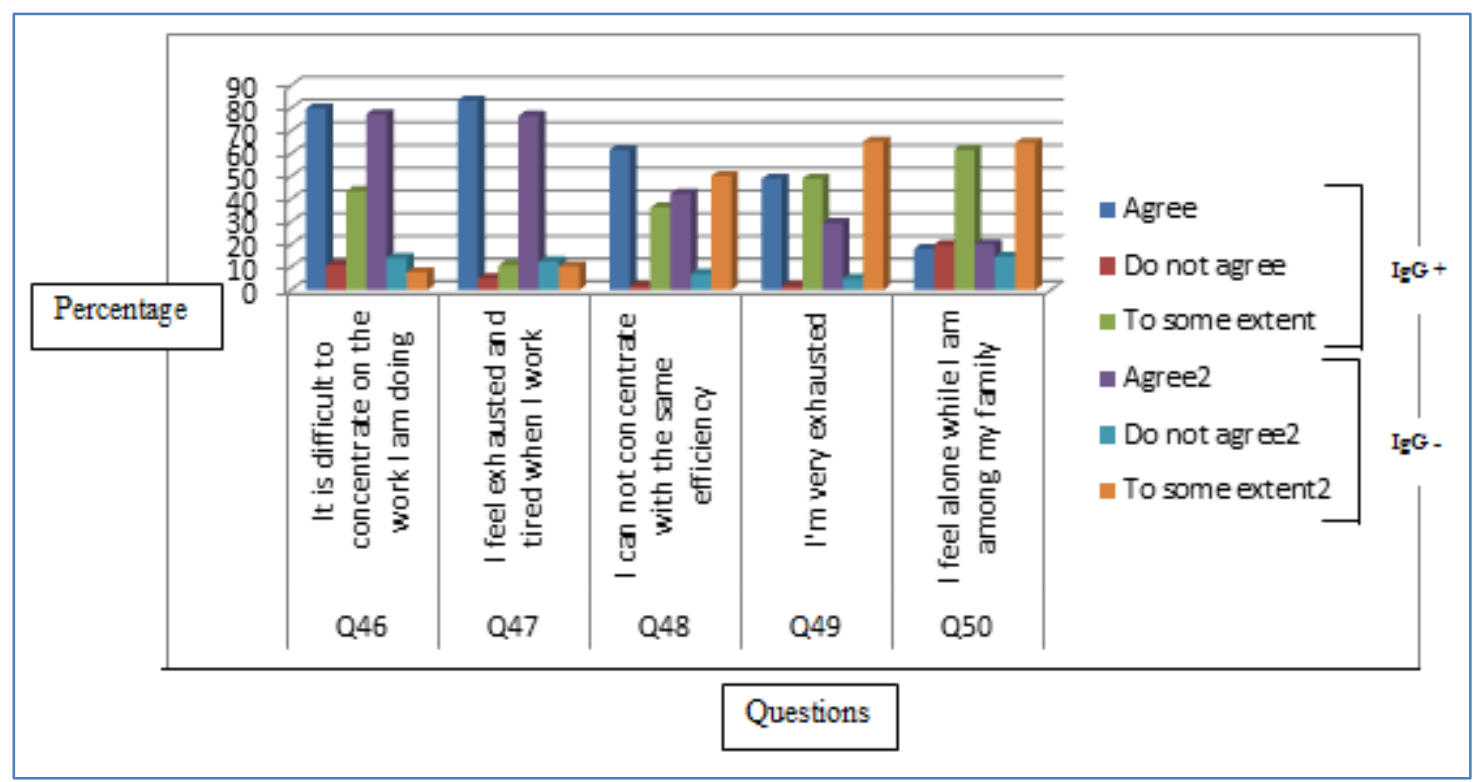

Figure 4. $4^{\text {th }}$ dimension: Exhaustion and poor concentration (no. of question 5)

Figure 4 shows the percentages of the different answers of the fourth dimension questions among IgG positive and IgG negative ELISA results. The results of dimension four showed that 3 out of 5 questions were with no relation with the latent $T$. gondii ( $p$ $>0.05$ ), while question 48 (I cannot concentrate with the same efficiency) and question 49 (I'm very exhausted) showed a close association with the toxoplasmosis IgG positive ELISA group $(p<0.05)$. Besides, the reduction in damage avoidance, fear of 
Citation: Khalil Mohamed, et al., The Effectiveness of Toxoplasma gondii Infection in the Emotions of Pregnant Women in Makkah, Saudi Arabia. Australian Journal of Basic and Applied Sciences, 13(1): 1-8. DOI: 10.22587/ajbas.2019.13.1.1

dying, decrease of death fear, and fear of the unknown (deterrents of suicide attempts) occasionally connected with suicide attempting, might be associated to a delicate tropism of the structures of the parasite involved in the neurocircuitry of fear and anxiety, such as the amygdale (Timothy et al., 2009).

In contrast to these studies, the current study has showed that no significant correlation between latent $T$. gondii infection and suicide attempts which suggest more work are needed to study these correlations.

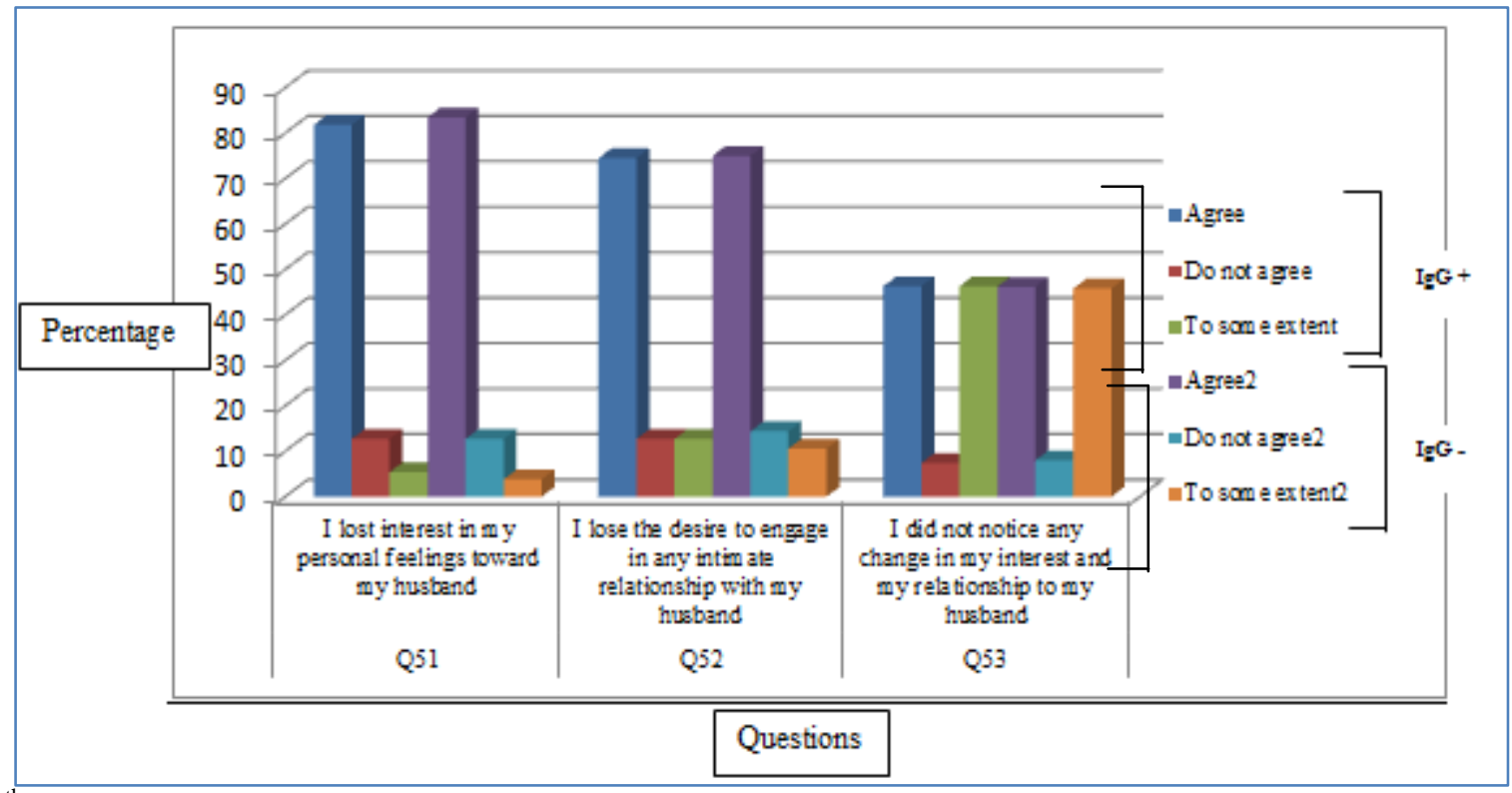

Figure 5. $5^{\text {th }}$ dimension: loss of interest in sex (no. of question 3)

Figure 5 shows the percentages of the different answers of the fifth dimension questions among IgG positive and IgG negative ELISA results. The results of dimension 5 which concentrated on loss of interest in sex, showed that all the questions were with no relationship with the latent $T$. gondii $(p>0.05)$. A recent study confirmed the reality of specific differences in desires, sexual behavior, and preferences between Toxoplasma-free and Toxoplasma-infected subjects (Flegr \& Kuba 2016).

Minor propensities for performance nonconventional sexual practices in the infected persons could be associated to their health status reduction (Flegr et al., 2016). However, these negative related were found even when controlled the covariates healthrelated (Flegr \& Kuba 2016). Some authors supposed that, in infected women decreased of personality factor could be responsible for the observed negative associations between toxoplasmosis and performing nonconventional sexual activities (Flegr et al., 2003; Novotná et al., 2005 \& Skallová et al., 2005)

In the current study, the results of last dimension questionnaire which deals with loss of interest in sex, have showed that all the questions were negatively responded indicated that no relation with the latent $T$. gondii infection $(p>0.05)$ and this result coincident with the previous publications.

\section{CONCLUSION}

According to the current study the effectiveness of chronic toxoplasmosis in pregnant women included feeling pessimistic about future, feeling not qualified in the life, and feeling has no benefit from her life but not thinking in suicide, insomnia, lost the concentration, and exhausted.

\section{ACKNOWLEDGEMENTS}

The authors would like to thank Institute of Scientific Research and Revival of Islamic Heritage at Umm Al-Qura University (project \# 43309004) for the financial support.

\section{REFERENCES}

Al-Harthi, S., M. Jamjoom, and H. Ghazi, 2006. Seroprevalence of Toxoplasma gondii among pregnant women in Makkah, Saudi Arabia. Umm Al-Qura University Journal for Science, Medicine and Engineering, 18: 217-227.

Almogren, A. 2011. Antenatal screening for Toxoplasma gondii infection at a tertiary care hospital in Riyadh, Saudi Arabia. Annals of Saudi Medicine, 31(6):569-72. doi: 10.4103/0256-4947.87090

Alvarado-Esquivel, C., L.F. Sanchez-Anguiano, J. Hernandez-Tinoco, L.O. Berumen-Segovia, Y.E. Torres-Prieto, S. EstradaMartinez, and O. Liesenfeld, 2016. Toxoplasma gondii Infection and Mixed Anxiety and Depressive Disorder: A CaseControl Seroprevalence Study in Durango, Mexico. Journal of Clinical Medicine Research, 8(7), 519-523. http://doi.org/10.14740/jocmr2576w. 
Citation: Khalil Mohamed, et al., The Effectiveness of Toxoplasma gondii Infection in the Emotions of Pregnant Women in Makkah, Saudi Arabia. Australian Journal of Basic and Applied Sciences, 13(1): 1-8. DOI: 10.22587/ajbas.2019.13.1.1

Arling, T.A., R H. Yolken, M. Lapidus, P. Langenberg, F.B. Dickerson, S.A. Zimmerman, and T.T. Postolache, 2009. Toxoplasma gondii Antibody Titers and History of Suicide Attempts in Patients With Recurrent Mood Disorders. Journal of Nervous and Mental Disease, 197(12):905-8. doi: 10.1097/NMD.0b013e3181c29a23

Ayi, I., A.Edu, and K. Apea-Kubi, 2009. Sero-epidemiology of toxoplasmosis amongst pregnant women in the greater Accra region of Ghana. Ghana Medical Journal, 43: 107-114.

Bansil, P.1., E.V Kuklina, S.F. Meikle, S. F. Posner, A.P. Kourtis, S.R. Ellington, and D. J. Jamieson, 2010. Maternal and fetal outcomes among women with depression. Journal of Womens' Health (Larchmt), 19(2):329-34. doi10.1089/jwh.2009.1387.

Carruthers, V.B., and Y. Suzuki, 2007. Effects of Toxoplasma gondii infection on the brain. Schizophrenia Bulletin, 33(3):745-51. DOI: $10.1093 / \mathrm{schbul} / \mathrm{sbm} 008$.

Cook, A. J., R. E. Gilbert, W. Buffolano, J. Zufferey, E. Petersen, P.A. Jenum, et al. and D. T. Dunn. 2000. Sources of Toxoplasma infection in pregnant women: European multicentre case-control study. European Research Network on Congenital toxoplasmosis. BMJ, 321: 142-147.

da Silva, M.G., M.C. Vinaud, and A.M. de Castro, 2015. Prevalence of toxoplasmosis in pregnant women and vertical transmission of Toxoplasma gondii in patients from basic units of health from Gurupi, Tocantins, Brazil, from 2012 to 2014. PLoS ONE, 10(11): e0141700. doi:10.1371/ journal.pone.0141700.

Flegr, J. and R. Kuba, 2016. The relation of Toxoplasma infection and sexual attraction to fear, danger, pain and submissiveness. Evolutionary Psychology,1-10, DOI: 10.1177/1474704916659746.

Flegr, J., J. Prandota, M. Sovicková, and Z.H. Israili, 2016. Toxoplasmosis - a global threat. Correlation of latent toxoplasmosis with specific disease burden in a set of 88 countries. PLoS ONE, 9(3): e90203. doi:10.1371/journal.pone.0090203.

Flegr, J., M. Preiss, J. Klose, J. Havlíček, M. Vitáková, and P. Kodym, 2003. Decreased level of psychobiological factor novelty seeking and lower intelligence in men latently infected with the protozoan parasite Toxoplasma gondii. Dopamine, a missing link between schizophrenia and toxoplasmosis? Biological Psychology, 63: 253-268. doi:10.1016/S0301-0511(03)00075-9

Flegr, J., S. Zitkova, P. Kodym, and D. Frynta, 1996. Induction of changes in human behavior by parasitic protozoan Toxoplasma gondii. Parasitology, 113:49-54.

Flegr, J., and J. Havlicek, 1999. Changes in the personality profile of young women with latent toxoplasmosis. Folia Parasitologica, 46:22-8.

Flegr, J., P. Kodym, , and V. Tolarová, 2000. Correlation of duration of latent Toxoplasma gondii infection with personality changes in women, 53, 57-68.

Flegr, J. 2007. Effect of Toxoplasma on human behavior. Schizophrenia Bulletin, 33:757-60. doi.org/10.1093/schbul/sb1074

Flegr, J., J. Havlícek, P. Kodym, and Z. Smahel, 2002. Increased risk of traffic accidents in subjects with latent toxoplasmosis: A retrospective case-control study. BMC Infectious Diseases; 2:11.

Flegr, J., J. Klose, M. Novotná, M. Berenreitterová, and J. Havlíček, 2009. Increased incidence of traffic accidents in Toxoplasmainfected military drivers and protective effect $\mathrm{RhD}$ molecule revealed by a large-scale prospective cohort study. BMC Infectious Diseases, 9:72. doi: 10.1186/1471-2334-9-72

Flegr, J., and I. Hardy, 1994. Influences of chronic toxoplasmosis on some human personality factors. Folia Parasitologica., 41: 122-26.

Fond, G., D. Capdevielle, Macgregor, A., J. Attal, A. Larue, M. Brittner, Ducasse, J., and P. Boulenger, 2013. Toxoplasma gondii: a potential role in the genesis of psychiatric disorders. Encephale, 39:38-43. doi: 10.1016/j.encep.

Ghazi, H.O., A.M. Telmesani, and M.F. Mahomed, 2002. TORCH agents in pregnant Saudi women. Medical Principals and Practice, 11(4):180-182. DOI: 10.1159/000065813

Groër, M. W., R.H. Yolken, X.W. Jason, J.W. Beckstead, D. Fuchs, S.S. Mohapatra, A. Seyfang, and T.T. Postolache, 2011. Prenatal depression and anxiety in Toxoplasma gondii-positive women. Gynecology, 204(5):433.e1-433e7. doi: 10.1016/j.ajog.2011.01.004

Heitman, B.B. and A.F. Irizarry, 1997. Recognition and management of toxoplasmosis. Nurse Practitioners, 22(9):75-86.

Jones, J., A. Lopes, and M. Wilson, 2003. Congenital toxoplasmosis. American Family Physician; 67: 2131-8.

Kaňková, Š., J. Šulc, K. Nouzová, K. Fajfrlík, D. Frynta, , and J. Flegr, 2007a. Women infected with parasite Toxoplasma have more sons. Naturwissenschaften, 94:122-127. DOI: 10.1007/s00114-006-0166-2

Kaňková, Š., P. Kodym, D. Frynta, R. Vavrinova, A. Kubena, and J. Flegr 2007b. Influence of latent toxoplasmosis on the secondary sex ratio in mice. Parasitology, 143:1709-19. DOI: 10.1017/S0031182007003253

Kocazeybek, B., Y. A. Oner, R. Turksoy, C. Babur, H. Cakan, and N. Sahip, , et al. 2009. Higher prevalence of toxoplasmosis in victims of traffic accidents suggest increased risk of traffic accident in Toxoplasma-infected inhabitants of Istanbul and its suburbs. Forensic Science International: Genetics, 187:103-108. DOI: 10.1016/j.forsciint.2009.03.007

Khalil, M., A. Bahathiq, N. Degnah, S. Basuni, A. Mahdi, A. Al Malki, and A. Babalghith, 2016 Detection of Toxoplasma gondii infection and associated risk factors among pregnant women in Makkah Al Mukarramah, Saudi Arabia, Asian Pacific Journal of Tropical Disease, 6(2):113-119. doi.org/10.1016/S2222-1808(15)60995-1

Ling,V. J., D. Lester, P. B. Mortensen, P. W. Langenberg, and T.T. Postolache, 2011. Toxoplasma gondii seropositivity and suicide rates in women. Journal of Nervous and Mental Disease, 199 (7):440-444. doi: 10.1097/NMD.0b013e318221416e.

Liu, Q., L. D. Singla, and H. Zhou, 2012. Vaccines against Toxoplasma gondii: status, challenges and future directions. Human Vaccines \& Immunotherapeutics, 8:1305-8. doi: 10.4161/hv.21006.

Liu, Q., Z. Wang, S. Huang, and X .Zhu, 2015. Diagnosis of toxoplasmosis and typing of Toxoplasma gondii. Parasites \& Vectors, 8:292. doi: 10.1186/s13071-015-0902-6.

Mann, J. 2003. Neurobiology of suicidal behaviour. Nature Reviews Neuroscience, 4 (10):819-828. DOI: 10.1038/nrn1220 
Citation: Khalil Mohamed, et al., The Effectiveness of Toxoplasma gondii Infection in the Emotions of Pregnant Women in Makkah, Saudi Arabia. Australian Journal of Basic and Applied Sciences, 13(1): 1-8. DOI: 10.22587/ajbas.2019.13.1.1

Miman, O., O.Y. Kusbeci, Aktepe, O.C., and Z. Cetinkaya, 2010. The probable relation between Toxoplasma gondii and Parkinson's disease. Neuroscience Letters, 475:129-131. doi: 10.1016/j.neulet.2010.03.057.

Montoya, J. G. and O. Liesenfeld, 2004. Toxoplasmosis. Lancet, 12; 363(9425):1965-76. DOI: 10.1016/S0140-6736(04)16412-X

Mocsny, N. 1992. Toxoplasmic encephalitis in the AIDS patient. Journal of Neuroscience Nursing, 24: 30-33.

Nissapatorn, V. 2007. Toxoplasmosis: A silent Threat in Southeast Asia. Research Journal of Parasitology, 2(1): 1-12. DOI: 10.3923/jp.2007.1.12

Novotná, M., J. Hanusova, J. Klose, M. Preiss, J. Havlicek, K. Roubalová, and J. Flegr, 2005. Probable neuroimmunological link between Toxoplasma and cytomegalovirus infections and personality changes in the human host. BMC Infectious Diseases, 5:54. doi: 10.1186/1471-2334-5-54.

Pessanha, T., M. de Carvalho, M. Pone, and S. Júnior, 2011. Diagnostic and therapeutic management of toxoplasmosis in pregnancy and the effect in the newborn. Revista Paulista de Pediatria, 29 (3):341-7. http://dx.doi.org/10.1590/S010305822011000300006.

Remington, J. S., \& J. L. Krahenbuhl, Immunology of Toxoplasma gondii. In: O’Reilly, A. J. (Ed.), Immunology of Human Infections, Part II. Plenum Publishing Corporation, New York, 1982, 327-371.

Robert-Gangneuxa, F., and M. Dardéc, 2012. Epidemiology of and Diagnostic Strategies for Toxoplasmosis. Clinical Microbiology Reviews, 25(2): 264-296. doi: 10.1128/CMR.05013-11.

Skallová, A., M. Novotná, P. Kolbeková, Z. Gasová, V. Veselý, M. Sechovská, , and J. Flegr, 2005. Decreased level of novelty seeking in blood donors infected with Toxoplasma. Neuroendocrinology Letters, 26: 480-486.

Sugden, K., T. Moffitt, L. Pinto, R. Poulton, B. Williams, and A. Caspi, 2016. Is Toxoplasma gondii infection related to brain and behaviour impairments in humans? Evidence from a population-representative birth cohort. Plos One, 11(2). doi.org/10.1371/journal.pone.0148435.

Tenter, A.M., A.R.Heckeroth, and L.M. Weiss, 2000. Toxoplasma gondii: from animals to humans. International Journal of Parasitology, 30: 1217-1258.

Timothy, A. A., Y.H. Robert, L. Manana, L. Patricia, D. B. Faith, Z.A. Sarah, B. Theodora, CA. Johanna, S. A. Debra, T.H. Leonardo, and P.T. Teodor, 2009. Toxoplasma gondii Antibody Titers and History of Suicide Attempts in Patients With Recurrent Mood Disorders. The Journal of Nervous and Mental Disease, 197(12):905-908. doi: 10.1097/NMD.0b013e3181c29a23.

Torrey, E. F., J J Bartko, Z.R. Lun, and R.H. Yolken, 2007. Antibodies to Toxoplasma gondii in patients with schizophrenia: A meta-analysis. Schizophrenia Bulletin; 33:729-736. doi: 10.1093/schbul/sbl050

Yagmur, F., S. Yazar, H.O. Temel, and M. Cavusoglu, 2010. May Toxoplasma gondii increase suicide attempt-preliminary results in Turkish subjects? Forensic Science International Genetics, 199 (1-3):15-17. doi: 10.1016/j.forsciint.

Yereli, K., I.C. Balcioğlu, and A. Özbilgin, 2006. Is Toxoplasma gondii a potential risk for traffic accidents in Turkey? Forensic Science International Genetics, 163(1-2):34-7. DOI: 10.1016/j.forsciint.2005.11.002 